\title{
A straightforward approach to multifunctional graphene
}

Matteo Andrea Lucherelli,, Jésus Raya, ${ }^{\ddagger}$ Konstantin F. Edelthalhammer, \& Frank Hauke, \& Andreas Hirsch, \& Gonzalo Abellán, $\dagger, \&, *$ Alberto Bianco, ${ }^{\sharp, *}$

\#University of Strasbourg, CNRS, Immunology, Immunopathology and Therapeutic Chemistry, UPR 3572, 67000 Strasbourg, France

‡ Membrane Biophysics and NMR, Institute of Chemistry, UMR 7177, University of Strasbourg, Strasbourg, France

\& Department of Chemistry and Pharmacy \& Joint Institute of Advanced Materials and Processes (ZMP), Friedrich-Alexander-Universität Erlangen-Nürnberg (FAU), Dr.-Mack-Straße 81, 90762, Fürth, Germany.

† Instituto de Ciencia Molecular (ICMol), Universidad de Valencia, Catedrático José Beltrán 2, 46980, Paterna, Valencia, Spain

Corresponding authors: gonzalo.abellan@uv.es, a.bianco@ibmc-cnrs.unistra.fr 


\section{Abstract}

Graphene has been covalently functionalized through a one-pot reductive pathway using graphite intercalation compounds, in particular $\mathrm{KC}_{8}$, with three different orthogonallyprotected derivatives of 4-aminobenzylamine. This novel multifunctional platform exhibits excellent bulk functionalization homogeneity and degree of addition while preserving the chemical functionalities of the organic addends through different protecting groups. We have employed (temperature-dependent) statistical Raman spectroscopy, X-ray photoelectron spectroscopy, magic angle spinning solid state ${ }^{13} \mathrm{C} N M R$, and a thermogravimetric analysis coupled with gas chromatography and mass spectrometry to unambiguously demonstrate the covalent binding and the chemical nature of the different molecular linkers. This work paves the way for the development of smart graphene based materials of great interest in biomedicine or electronics, and will serve as a guide in the design of new 2D multifunctional materials. 
Since its discovery, the chemical functionalization of graphene has been investigated with the purpose to tune its unique electrical properties and improve the functionalities of the material. ${ }^{[1]}$ The development of functional materials can be considered one of the great challenges on the chemistry of graphene, with the purpose to increase the performances of this $2 \mathrm{D}$ material. The reactivity of graphene has shown to be strongly correlated to the number of layers constituting each sheet, and it decreases dramatically with the increasing of the number of layers. ${ }^{[2,3]}$ For this reason, an extensive variety of organic reactions has been reported in the literature for monolayered graphene, ${ }^{[3,4]}$ but not all these reactions can be applied satisfactory for a scale-up production from bulk graphene. In this context, the reduction of graphite using alkaline metals yielding graphite intercalation compounds (GICs), followed by the quenching of the intermediately generated graphenides ${ }^{[5]}$ with electrophiles, is an appropriate alternative to enhance the functionalization degree in bulk graphene dispersions. ${ }^{[6-8]}$ The reactivity of intercalated graphenide species is higher respect to few layer graphene, and strongly dependent on the ratio between carbon and metal atoms, leading to different degree of functionalization. ${ }^{[8]}$ Besides these fundamental studies, one of the most important synthetic challenges in graphene chemistry that remains to be addressed is to develop a straightforward approach for the bulk synthesis of graphene functionalized with several molecules (i.e. multifunctionalization). Here we have investigated the multifunctionalization in one-step reaction of bulk graphene, taking advantage of the reductive route using potassium-intercalated $\mathrm{KC}_{8} \mathrm{GICs} .{ }^{[7]}$ This approach allowed us to obtain for the first time a multifunctional platform with a high level of functionalization and in elevate quantities, suitable for the development of smart graphene-based materials. Thanks to the cross-use of several high-end complementary techniques including statistical Raman spectroscopy (SRS), X-ray photoelectron spectroscopy (XPS), magic-angle-spinning solid state NMR (MAS-NMR), thermogravimetric analysis coupled with gas chromatography and mass spectrometry (TGA/GC/MS), and Kaiser test, we were able to recognize and quantify the presence of the three different functional groups introduced on the surface of graphene. Our results demonstrate that it is possible to synthesize in one pot a multifunctional graphene derivative with three different orthogonally-protected derivatives of 4aminobenzylamine, while obtaining a high degree of addition and excellent bulk homogeneity. 
Our approach to the preparation of a multifunctional graphene (mf-G) started with the synthesis of three different orthogonally-protected derivatives of 4-aminobenzylamine (see $\mathrm{SI}),{ }^{\left[{ }^{[9]}\right.}$ followed by the synthesis and isolation of the corresponding diazonium salts (Scheme 1). With these reagents in hands, we then intercalated pristine natural graphite (synthetic spherical graphite, SGN18) with metallic potassium, ${ }^{[7]}$ yielding $\mathrm{KC}_{8}$, an intermediate reduced form of graphite. Intercalated graphite is highly reactive respect to exfoliated few layers graphene and its preparation requires inert atmosphere and complete absence of water ${ }^{[8,10,11]} \mathrm{KC}_{8}$ was sonicated in dry and degassed THF by tip sonication for $5 \mathrm{~min}$ to favour the almost complete exfoliation of graphene in monolayers, leading to a graphenide dispersion. ${ }^{[5,12,13]}$ One equivalent (constituted of $1 / 3$ of each derivative) of the diazonium salts was mixed with $\mathrm{KC}_{8}$ and reacted for $2 \mathrm{~d}$ at $\mathrm{rt}$. After this time, benzonitrile was added under argon atmosphere, as scavenger for the residual negative charges to avoid side reactions, such as oxidation with atmospheric $\mathrm{O}_{2}$ during the work-up, due to an incomplete charge-quenching of the graphenides. ${ }^{[8,13,14]}$ The material was then washed with dichloromethane, ethanol and water, to remove the organic species present in the mixture.

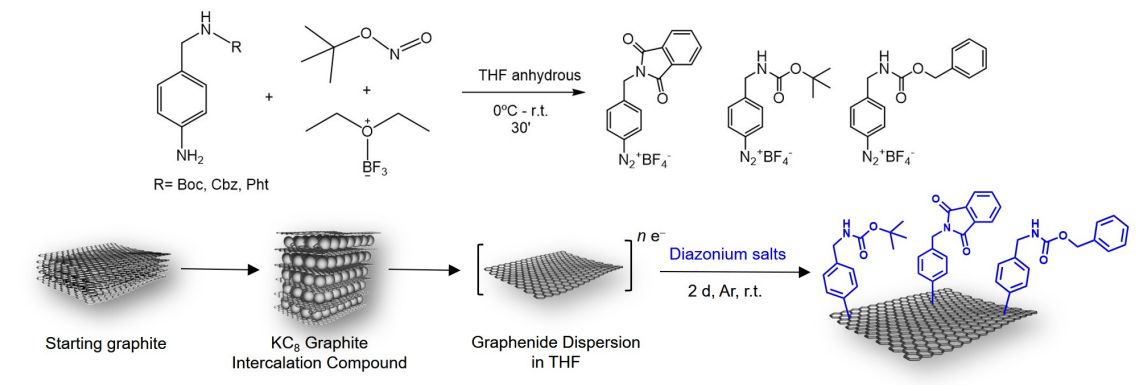

Scheme 1: Synthesis of the diazonium salt derivatives and functionalization of $\mathrm{KC}_{8}$. Pht, phtalimide; Boc, tert-butyloxycarbonyl; Cbz, benzyloxycarbonyl.

The evaluation of the functionalization of graphene started with statistical Raman spectroscopy (SRS). The analysis of the average Raman spectra revealed an increasing of the intensity of the $D$ band respect to the $G$ band of the bulk graphite $\left(I_{D} / I_{G}\right.$ ratio 0.1$)$, and the appearance of the $D^{\prime}$ band. Moreover, the typical 2D band at $\sim 2690 \mathrm{~cm}^{-1}$ is overlapped by the appearing of a $D+D^{\prime}$ band at $\sim 2900 \mathrm{~cm}^{-1}$. This phenomenon is in agreement with the data reported in the literature and is as consequence of the $A_{1 \mathrm{~g}}$ mode-breathing vibrations of sixthe $\mathrm{sp}^{2}$ graphene lattice.... 
membered $\mathrm{sp}^{2}$ carbon rings, which becomes Raman active after the symmetry of the nearby lattice is reduced ( $\mathrm{sp}^{2}$ carbons are converted to $\mathrm{sp}^{3}$ hybridization) by the covalent functionalization with the diazonium salts. ${ }^{[8,15-17]}$ The calculation of the intensity $I_{D} / I_{G}$ distribution functions between the $\mathrm{D}$ band and the $\mathrm{G}$ band resulted in a value of 1.3 (Figure 1a). The narrow statistical distribution of the $I_{D} / I_{G}$ shows a residual contribution of pristine unreacted graphite and high homogeneity of the entire bulk functionalization ( $H_{\text {bulk, }}$ Figure $\left.1 \mathrm{~b}\right){ }^{[18]}$
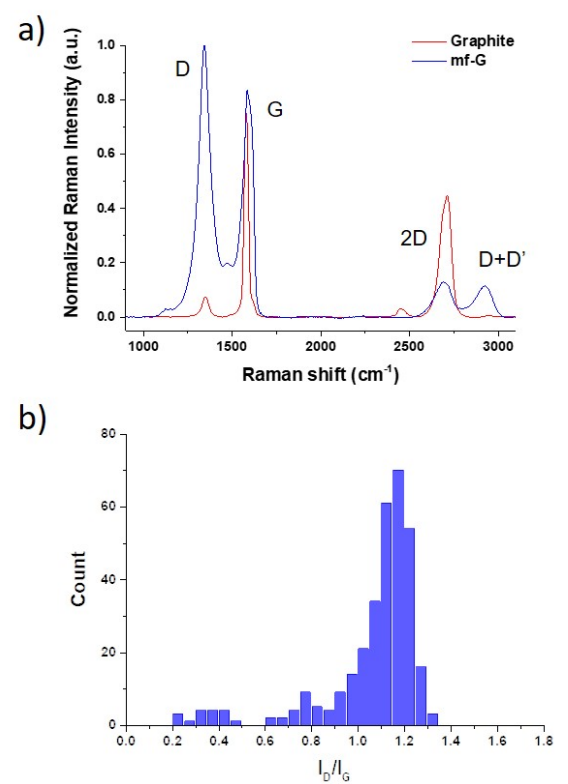

Figure 1: a) Comparison between mean Raman spectra (500 single-point spectra) of graphite and $\mathrm{mf}-\mathrm{G} ; \mathrm{b}$ ) Distribution of $\mathrm{I}_{\mathrm{D}} / \mathrm{I}_{\mathrm{G}}$ ratio from the Raman statistical analysis.

To assure that the drastic conditions of the reactions on graphene and that the functionalization do not affect the morphology of the layers, mf-G was dispersed in DMF by bath sonication and observed by transmission electron microscopy (TEM), confirming the typical shape of 2D materials (Figure S1). Subsequently, we investigate, through temperature-dependent statistical Raman spectroscopy (T-SRS), the thermal evolution of the material between 25 and $450{ }^{\circ} \mathrm{C}$. The $I_{D} / I_{G}$ ratio, slowly diminish until $200{ }^{\circ} \mathrm{C}$ and then starts to decrease dramatically reaching mean $\mathrm{I}_{\mathrm{D}} / \mathrm{I}_{\mathrm{G}}$ values of 0.45 at $425{ }^{\circ} \mathrm{C}$ (Figure 2a). This phenomenon is due to the removal of the functional groups from the surface of graphene and corresponds to the rehybridization from $\mathrm{sp}^{3}$ to $\mathrm{sp}^{2}$ of the carbon atoms. In addition, it is 
also remarkable the increase of the $2 D$ band and the decrease of the $D+D^{\prime}$ band, confirming that the defunctionalization process of the diazonium salt derivatives was occurring (Figure $2 \mathrm{~b})$. The observation of the detachment of covalently bound moieties was corroborated by the thermogravimetric analysis (TGA). The weight loss profile (Figure 2a) is stable until 220 ${ }^{\circ} \mathrm{C}$, temperature above which the drastic weight loss begun. To well understand the slight difference on the defunctionalisation temperature observed between the two abovementioned techniques, we must consider that the warming-up ramp programs present small differences. TGA program warm-up the samples at $10^{\circ} \mathrm{C} / \mathrm{min}$ uniformly, while during Raman analysis the sample is warmed-up in successive steps, keeping stable the settled temperature during the scanning time. We can then affirm that the overall defunctionalization starts between $200^{\circ}$ and $220^{\circ} \mathrm{C}$. Moreover, the observed weight loss of $\sim 15 \%$ is in good agreement with the $I_{D} / I_{G}$ ratio value of 1.3 , corresponding to a high degree of functionalization of graphene. ${ }^{[8]}$ In addition, these results suggest the absence of secondary products, in good agreement with combined thermogravimetry gas chromatography and mass spectrometry analysis (vide infra).

a)

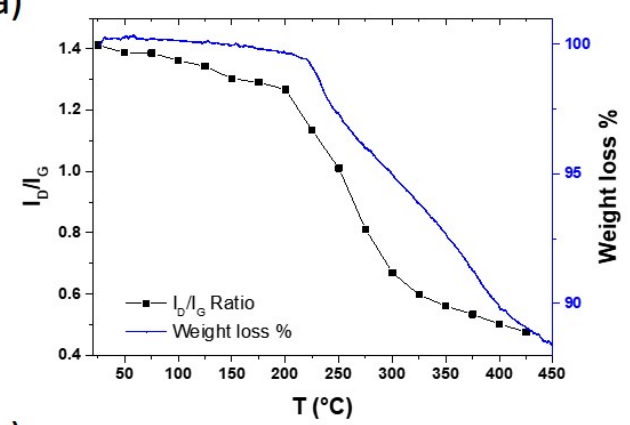

b)

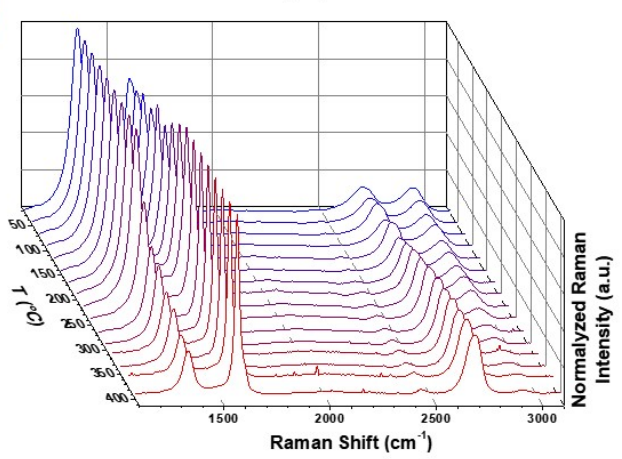


Figure 2: Temperature-dependent statistical Raman spectroscopy (TSRS) of $\mathrm{mf}-\mathrm{G}$ in the temperature region between 25 and $450{ }^{\circ} \mathrm{C}$. a) $\mathrm{I}_{\mathrm{D}} / \mathrm{I}_{\mathrm{G}}$ ratio plotted respect to temperature and TGA analysis of mf-G. b) Means of the Raman temperature depending spectra.

The high reactivity of $\mathrm{KC}_{8}$ species was compared to the neutral route, i.e. the reaction to form mf-G performed starting from pristine few layers graphene exfoliated in NMP (see SI for the details). Graphene was reacted with a mixture of the same diazonium salts used to modify $\mathrm{KC}_{8}$ following two different paths, one forming in situ the salts (mf- $\mathrm{G}_{\text {situ}}$ ) using isopentyl nitrite, and the second using the preformed and well-characterised diazonium salts ( $m f-G_{p r e}$ ). As reported by the group of Hirsch, ${ }^{[7]}$ the functionalization of few layer graphene with diazonium salts requires a high ratio between graphene and the functional molecules $(\sim$ 1:6 eq.) to obtain materials with an elevated degree of functionalization. Besides, the use of high amount of reactive species is leading to side reactions, such as modification of the introduced functional groups or quenching of the reactive species by radical recombination. ${ }^{[19]}$ Following the previous protocol, we performed the reaction in a ratio of 1:4 and 1:6 between graphene and the diazonium salts, obtaining a much lower degree of functionalization than in the case of $\mathrm{KC}_{8}$. The Raman analysis allowed us to measure an $\mathrm{I}_{\mathrm{D}} / \mathrm{I}_{\mathrm{G}}$ mean ratio of 0.36 and 0.41 for $\mathrm{mf}-\mathrm{G}_{\text {situ }}$ and $\mathrm{mf}-\mathrm{G}_{\text {pre, }}$ respectively (Figure S2). Then, TGA analysis showed for both reactions a weight loss value of $\sim 7 \%$ (Figure S3). Both techniques confirmed the low degree of functionalization of these materials, which was not satisfactory and suitable for further applications of $\mathrm{mf}-\mathrm{G}$.

The data obtained from Raman and TGA are relative to the quantity of defects introduced on graphene surface but do not provide information about their chemical nature. To verify the effective functionalization with the different diazonium salt derivatives, other techniques were applied. First, the presence of $\mathrm{N}$ element was investigated by X-ray photoelectron spectroscopy (XPS). The atomic percentage composition of $\mathrm{mf}-\mathrm{G}$ was measured, revealing a ratio between $\mathrm{C}, \mathrm{O}$ and $\mathrm{N}$ of $86.2 \%, 10.8 \%$ and $3.0 \%$ for the $\mathrm{mf}-\mathrm{G}$, respectively, and of $94.5 \%, 5.2 \%$ and $0.35 \%$, respectively, for graphene after the initial potassium intercalation. The increment of $\mathrm{N}$ is the first advice that the increase of the defects observed by Raman spectroscopy is related to the grafting of the new functional groups and not due to a simple oxidation of graphene. In order to obtain a direct and unique confirmation of the presence of the functional groups on graphene, the Kaiser test was performed on mf-G. ${ }^{[20]}$ Each protecting group was selectively removed (see SI for details). The obtained results of 76, 42 
and $46 \mu \mathrm{mol} / \mathrm{g}$ for Boc, Cbz and Pht protected amines, respectively, are the proof of the presence of the expected functional groups grafted on graphene. The abundance of Boc protected amine respect to $\mathrm{Cbz}$ and Pht is due to the reactivity of the species, similarly to the data that we previously reported (Boc $>\mathrm{Pht}>\mathrm{Cbz}) .{ }^{[9]}$ To further explore the presence of the functional groups on the graphene structure, we analysed mf-G using MAS-NMR. ${ }^{13} \mathrm{C}$ solid state NMR on single graphene sheets is rather challenging as it faces specific issues lying on high electric conductivity. ${ }^{[21,22]}$ Electric conductivity causes probe tuning problems, ${ }^{[21,23]}$ and very often gives rise to extremely wide lines ${ }^{[24]}$ that may become undetectable or indistinguishable from probe background signal. We recorded here a series of DP/MAS (Direct Polarization/Magic Angle Spinning) on a $750 \mathrm{MHz}$ spectrometer with a spin-echo sequence ${ }^{[25]}$ that cures the background signal problem and gives flat baselines and undistorted line shapes. We performed and compared the spectra of graphene after the intercalation of potassium, of $\mathrm{KC}_{8}$ mixed with the starting reagent $\left(\mathrm{G}_{\text {mixed }}\right)$ and of the final $\mathrm{mf}$ $\mathrm{G}$. In the case of the $\mathrm{KC}_{8}$, as expected for graphene, we had to finely mix it with an insulating powder $\left(\mathrm{SiO}_{2}\right.$ in a ratio of 1:4 graphene to $\left.\mathrm{SiO}_{2}\right)$ to avoid the sample behaves as a bulk conductor $^{[21]}$ and to achieve correct probe tuning and fast sample spinning. In addition, no clear signal of functional carbon atoms was detected (Figure 3, bottom), which may be an indication of $\mathrm{KC}_{8}{ }^{13} \mathrm{C}$ nuclei strongly affected by their interactions with conductive electrons. ${ }^{[22,26]}$ When compared with the residual probe background signal (Figure 3 , bottom) very small contribution could be ascribed to $\mathrm{KC}_{8}$, while most of the spectral bands are resulting from the probe/spinner system. Clearly both signals, probe background and $\mathrm{KC}_{8}$, are much wider than the filter size used in the spin-echo sequence and they were mostly removed together by the filter. As first evidence of the occurred modification of graphene surface, the electrical conductivity in $\mathrm{mf}-\mathrm{G}$ resulted almost negligible respective to $\mathrm{KC}_{8}$. The probe was perfectly tuning, and the spectrum was collected without mixing with $\mathrm{SiO}_{2}$. Three peaks, at 7.0, 49.0 and 103 ppm (Figure 3, top) with rather wide (5 kHz) but detectable lines, are visible. This clearly implies a structural modification that cannot be due to simple stacking of reagents on graphene surface: the case is given in $G_{\text {mixed }}$ spectrum (Figure S3, top) where the pure reagents (Figure S3, bottom) mostly keep their chemical shifts (30.0 ppm, $81.3 \mathrm{ppm}$ and $146.2 \mathrm{ppm}$ ) together with a small line widening $(50 \mathrm{~Hz})$, probably due to magnetic susceptibility effects arising from $\mathrm{KC}_{8}$. It is important to note that mf-G presents the same peak series than $G_{\text {mixed, }}$ but with big differences in chemical shift. 
Such difference in mf- $G$ and $G_{\text {mixed }}$ spectral features strongly pleads in favour of the covalent functional groups grafting. Indeed, these kind of unexpected variations in chemical shifts are not uncommon in graphene systems and have been ascribed to magnetic susceptibility effect, ${ }^{[23]}$ knight shift effects or to the nucleus independent chemical shift. ${ }^{[27]}$ It has also been recently shown that the presence of local structural distortions in graphene can affect the values of the ${ }^{13} \mathrm{C}$ isotropic shielding constant leading to additional shifts in the same orders than those found for mf-G. ${ }^{[28]}$ Based on our experience, we hypothesized that the peak at $7.0 \mathrm{ppm}$ can be assigned to the aliphatic carbon atoms, likely corresponding to the $\mathrm{CH}_{3}$ of Boc. The peak at $49.0 \mathrm{ppm}$ can correspond to the carbon atoms bound to oxygen and the peak at $103 \mathrm{ppm}$ could be related to carbon atoms arranged in aromatic rings. This last assignment is corroborated by the hypothesis that with the increasing of defects on graphene surface, the aromatics rings could result as more localized on the structure.

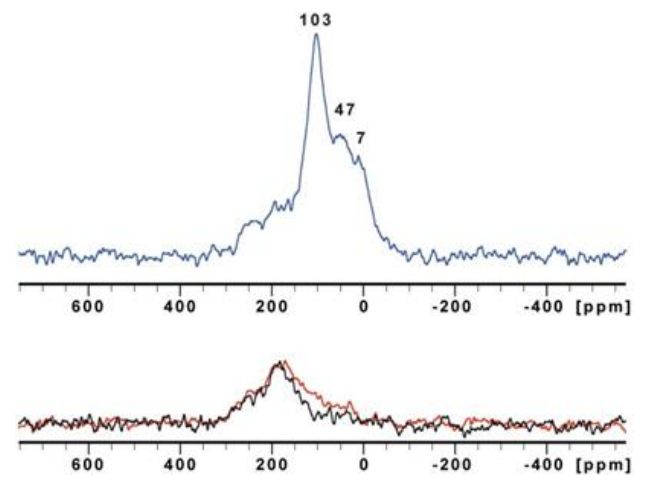

Figure 3: ${ }^{13} \mathrm{C}$ SS NMR spectra of mf-G and $\mathrm{KC}_{8}$. Top: DP spin-echo on mf-G. Peaks at 7, 47 and $103 \mathrm{ppm}$ superimpose with probe background signal (bottom, black). Bottom: $\mathrm{KC}_{8}$ (red) and probe background signal (black) in the same conditions.

Lastly, to face the problem of the direct identification of the functional groups grafted onto graphene, we analyzed the $\mathrm{mf}-\mathrm{G}$ with a technique based on the coupling between TGA, gas chromatography and mass spectroscopy (TG-GC-MS). ${ }^{[8]}$ We collected and eluted by GC the molecules and fragments in gas phase generated by the TGA at different temperature of the analysis, to evaluate the composition of the samples. Not all the $m / z$ fragments collected could be assigned with certainty to a precise functional group. But, GC allowed the separation and the mass analysis of each fragment developed during the TGA process, 
enhancing the interpretation of the MS spectra. From the chromatogram of the gas injected at the temperature of $320^{\circ} \mathrm{C}$ (Figure S5), evidence of the presence of Boc protecting group was derived from the characteristic mass signal at $\mathrm{m} / \mathrm{z} 57$ eluted with the peak at $3.57 \mathrm{~min}$. Moreover, two different peaks at 8.60 and 9.72 min were detected. From the chromatogram of the gas injected at $200{ }^{\circ} \mathrm{C}$ is it possible to predominantly observe the peak at $4.37 \mathrm{~min}$, corresponding to THF adsorbed on $\mathrm{mf}-\mathrm{G}$ (Figure S6).

The peak at 8.60 min contains mass fragments with $\mathrm{m} / \mathrm{z}$ ratio of 51,77 and 105 (Figure $\mathrm{S} 7 \mathrm{~b}$ ). Comparing our MS spectra with a library of spectra, we could observe that these fragments match with the spectra of phenylglyoxal. This molecule is a structural isomer of the fragment of $\mathrm{Cbz}$ protecting group. To verify if the observed peak was really related to $\mathrm{Cbz}$ fragmentation, we prepared a control experiment consisting of functionalised graphene with only the $\mathrm{Cbz}$ diazonium salt derivate, observing the appearance of the same peak at 8.60 min, thus confirming the presence of this functional group. Finally, the peak at $9.72 \mathrm{~min}$ showed fragments with $\mathrm{m} / \mathrm{z}$ of 77 and 108 (Figure S7c), matching with the MS spectra of benzyl alcohol. We hypothesised that this fragment could be generated from a rearrangement of the structure of the phenyl protecting group during the TGA process, after losing a NHCO group. Thanks to the coupling between TGA, GC and MS it was possible to observe fragments with similar $m / z$ at different retention times and assign each one of these to the correspondent functional group on graphene. In light of these results we have direct evidence about the presence of the occurred functionalization by the three diazonium salts. In summary, we developed a one-pot multifunctionalization of graphene with three different orthogonally-protected derivatives of 4-aminobenzylamine obtaining high degrees of addition and bulk homogeneity. We followed a reductive route using $\mathrm{KC}_{8}$ graphite intercalation compound as activated precursor and an equimolecular mixture of different diazonium salts as electrophiles. Overall, the combination of advanced analytical tools allowed for the detailed identification of the covalently bound organic addends, proving the versatility of this synthetic approach. This work may serve as a guideline for the design of 2D multifunctional materials of great interest in biomedicine, electronics, sensing, or energy storage and conversion.

\section{Acknowledgements}


G.A. thanks the financial support from the EU (ERC Starting Grant 2D-PnictoChem 804110), the Spanish MINECO (Unit of Excellence "Maria de Maeztu" MDM-2015-0538), the Generalitat Valenciana (CIDEGENT/2018/001 grant), the DFG (FLAG-ERA AB694/2-1; SFB 953 "Synthetic Carbon Allotropes", project A1) and the Cluster of Excellence Engineering of Advanced Materials. A.B. acknowledges financial support from the ANR (ANR-15-GRFL-000105, G-IMMUNOMICS project), the CNRS, and the LabEx project Chemistry of Complex Systems (ANR-10-LABX-0026_CSC).

\section{Reference}

[1] Y. Hu, X. Su, Adv. Graphene Sci. 2013, 161-189.

[2] F. M. Koehler, A. Jacobsen, K. Ensslin, C. Stampfer, W. J. Stark, Small 2010, 6, 1125-1130.

[3] A. Criado, M. Melchionna, S. Marchesan, M. Prato, Angew. Chemie - Int. Ed. 2015, 54, 1073410750.

[4] L. Gan, D. Zhang, X. Guo, Small 2012, 8, 1326-1330.

[5] G. Bepete, E. Anglaret, L. Ortolani, V. Morandi, K. Huang, A. Pénicaud, C. Drummond, Nat. Chem. 2017, 9, 347-352.

[6] A. Pénicaud, C. Drummond, Acc. Chem. Res. 2013, 46, 129-137.

[7] J. M. Englert, C. Dotzer, G. Yang, M. Schmid, C. Papp, J. M. Gottfried, H. P. Steinrück, E. Spiecker, F. Hauke, A. Hirsch, Nat. Chem. 2011, 3, 279-286.

[8] G. Abellán, M. Schirowski, K. F. Edelthalhammer, M. Fickert, K. Werbach, H. Peterlik, F. Hauke, A. Hirsch, J. Am. Chem. Soc. 2017, 139, 5175-5182.

[9] C. Ménard-Moyon, C. Fabbro, M. Prato, A. Bianco, Chem. - A Eur. J. 2011, 17, 3222-3227.

[10] F. Hof, R. A. Schäfer, C. Weiss, F. Hauke, A. Hirsch, Chem. - A Eur. J. 2014, 20, 16644-16651.

[11] K. C. Knirsch, R. A. Schäfer, F. Hauke, A. Hirsch, Angew. Chemie - Int. Ed. 2016, 55, 5861-5864.

[12] E. M. Milner, N. T. Skipper, C. A. Howard, M. S. P. Shaffer, D. J. Buckley, K. A. Rahnejat, P. L. Cullen, R. K. Heenan, P. Lindner, R. Schweins, J. Am. Chem. Soc. 2012, 134, 8302-8305.

[13] S. A. Hodge, D. J. Buckley, H. C. Yau, N. T. Skipper, C. A. Howard, M. S. P. Shaffer, Nanoscale 2017, 9, 3150-3158.

[14] P. Vecera, J. Holzwarth, K. F. Edelthalhammer, U. Mundloch, H. Peterlik, F. Hauke, A. Hirsch, Nat. Commun. 2016, 7, 12411.

[15] A. Eckmann, A. Felten, I. Verzhbitskiy, R. Davey, C. Casiraghi, Phys. Rev. B - Condens. Matter Mater. Phys. 2013, 88, 1-11.

[16] L. G. Cançado, A. Jorio, E. H. M. Ferreira, F. Stavale, C. A. Achete, R. B. Capaz, M. V. O. Moutinho, A. Lombardo, T. S. Kulmala, A. C. Ferrari, Nano Lett. 2011, 11, 3190-3196.

[17] S. Niyogi, E. Bekyarova, M. E. Itkis, H. Zhang, K. Shepperd, J. Hicks, M. Sprinkle, C. Berger, C. N. Lau, W. A. Deheer, et al., Nano Lett. 2010, 10, 4061-4066.

[18] J. M. Englert, P. Vecera, K. C. Knirsch, R. A. Schäfer, F. Hauke, A. Hirsch, ACS Nano 2013, 7, 5472-5482.

[19] J. Greenwood, T. H. Phan, Y. Fujita, Z. Li, O. Ivasenko, W. Vanderlinden, H. Van Gorp, W. Frederickx, G. Lu, K. Tahara, et al., ACS Nano 2015, 9, 5520-5535.

[20] E. Kaiser, R. L. Colescott, C. D. Bossinger, P. I. Cook, Anal. Biochem. 1970, 34, 595-598.

[21] M. Toyoda, T. Tsumura, B. Tryba, S. Mozia, M. Janus, A. W. Morawski, M. Inagaki, Chem. Phys. Carbon Vol. 31 2012, 171-368.

[22] M. A. Vieira, G. R. Gonçalves, D. F. Cipriano, M. A. Schettino, E. A. Silva Filho, A. G. Cunha, F. G. Emmerich, J. C. C. Freitas, Carbon N. Y. 2016, 98, 496-503.

[23] J. C. C. Freitas, F. G. Emmerich, G. R. C. Cernicchiaro, L. C. Sampaio, T. J. Bonagamba, Solid 
State Nucl. Magn. Reson. 2001, 20, 61-73.

[24] M. A. Vieira, C. M. R. Frasson, T. L. G. Costa, D. F. Cipriano, M. A. Schettino, A. G. Cunha, J. C. C. Freitas, Quim. Nova 2017, 40, 1164-1171.

[25] E. L. Hahn, Phys. Rev. 1950, 80, 580-594.

[26] J. M. Franck, S. Han, Methods Enzymol. 2019, 615, 131-175.

[27] L. B. Casabianca, J. Phys. Chem. A 2016, 120, 7011-7019.

[28] F. A. L. De Souza, A. R. Ambrozio, E. S. Souza, D. F. Cipriano, W. L. Scopel, J. C. C. Freitas, J. Phys. Chem. C 2016, 120, 27707-27716. 
Graphical Abstract

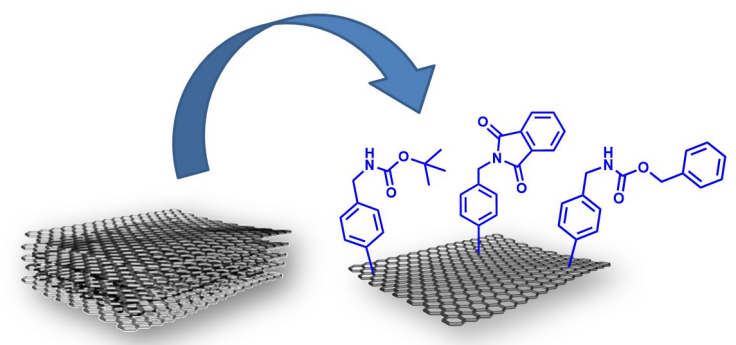

Multifunctionalized graphene can be easily obtained from graphite intercalation compounds. 